Ann. Génét. Sẹl. anim., I971, 3 (3), 28 I-293.

\title{
DISTRIBUTIONS DE FRÉQUENCES ; INTERPRÉTATION DU DÉTERMINISME GÉNÉTIQUE DES CARACTÈRES QUANTITATIFS ET RECHERCHE DE GENES « MAJEURS »: DONNÉES EXPÉRIMENTALES
}

\author{
P. MÉRAT
}

avec la collaboration technique de L. Durand et A. Bordas

Laboratoive de Génétique factorielle,

Centre national de Recherches zootechniques, I.N.R.A., 78-Jouy-en-Josas

\section{RÉSUMÉ}

La présence de gènes à effet important a été recherchée dans plusieurs populations pedigree de volailles de Jouy-en-Josas et de la Station du Magneraud, et pour divers caractères quantitatifs dont la distribution sur l'ensemble de chaque population étudiée ne s'écarte pas considérablement de la normalité : poids corporel, ponte, caractères des œufs, poids d'organes. Un test proposé antérieurement (MÉrat, 1968) est utilisé : examen de la forme (symétrie, kurtosis) des distributions des écarts aux moyennes de familles séparément parmi les familles à plus grande et à plus petite variance observée, lorsqu'une hétérogénéité de ces variances intra-familles a été préalablement mise en évidence.

Dans une forte proportion des cas, les variances intra-familles sont très significativement hétérogènes. D'autre part, la distribution des écarts aux moyennes de familles est assez souvent non-normale pour les familles à grande variance, et pratiquement jamais pour les autres, dans les limites de sensibilité du test. Les écarts à zéro des coefficients de symétrie et de kurtosis sont plus fréquemment significatifs et plus extrêmes dans le groupe des "grandes variances "; en outre, les coefficients de kurtosis sont, pour leur majorité, négatifs.

L'ensemble de ces résultats suggère l'existence, pour une porportion notable des cas, d'un nombre limité de gènes ou régions chromosomiques rendant compte d'une part importante de la variance génétique totale. Ils sont donc un encouragement à poursuivre cette recherche.

\section{INTRODUCTION}

On a cherché depuis longtemps à isoler, dans un caractère à variation continue, des "facteurs " génétiques à effet individuel appréciable, loci à proprement parler ou régions chromosomiques d'étendue limitée. Les solutions proposées sont de deux types. 
Le premier est l'utilisation de gènes "marqueurs " pour localiser certains des facteurs recherchés. D'assez nombreux exemples en sont connus, chez des végétaux, et, surtout, chez la drosophile (en particulier EAST I9I6; SAX, I923; WRIGHT, I927; Thoday, i96r; Spicketr, I963; Thoday, Gibson et Spickett, I964; Spickett et Thoday, I966). Parmi les animaux domestiques, le lapin (CASTLE, I940) et la poule en fournissant des illustrations, les marqueurs étant, dans cette dernière espèce, des gènes de groupe sanguin (Gilmour, I960) ou à effet visible (MERAT, I967 $a$ et $b$ ).

Le second type de solutions est de mise en œuvre plus difficile, puisqu'il correspond au cas où l'on ne dispose pas de marqueurs, cas le plus fréquent dans les espèces domestiques.

En général (I), les méthodes proposées reposent sur la comparaison de lignées parentes, supposées de préférence homozygotes pour les gènes importants recherchés, avec leurs $F_{1}, F_{2}$, back-crosses, le cas échéant $F_{3}$. Le premier aspect développé a été l'estimation d'un nombre minimum de loci en cause : CASTLE (I92I $a$ et $b$ ), WRIGHT (I952). Celle-ci est obtenue, sous certaines hypothèses restrictives, à partir de la différence moyenne entre lignées parentales, et de 1'excès de variance de la $F_{2}$ sur la $F_{1}$. Diverses corrections sont apportées, pour le cas de dominance ou pour celui de linkage (SEREBrovsky, I928; Dempster et SNyder, I950). Charles et Goodwin (I943) transposent cette méthode pour l'estimation d'un nombre minimum de loci influant à la fois sur deux caractères.

Les auteurs cités indiquent eux-mêmes le caractère imprécis des estimations proposées. Celles-ci représentent une évaluation minimum, supposant réalisées 1'homozygotie des lignées parentes, l'absence d'interaction entre loci, l'égalité de la variance "environnement " entre les générations comparées. En outre, le postulat tout théorique de l'égalité des effets des différents gènes indique bien qu'il ne peut s'agir que de suggérer un ordre de grandeur quant à leur nombre.

On comprend ainsi que l'on ne se soit pas préoccupé de chercher un intervalle de confiance aux estimations correspondantes. De la sorte, elles ne répondent pas exactement à la question que nous nous posons, à savoir de tester la présence de " facteurs » à effet individuel appréciable, sans chercher dès l'abord à évaluer leur nombre.

Dans un ordre d'idées un peu analogue, FISHER, IMMER et TEDIN (I932) et MATHER (I949) se servent des déviations de dominance évaluées par l'observation des $F_{1}$ et $F_{2}$ pour estimer, ici encore, $u$ nombre minimum de loci. responsables de la variation d'un caractère. Ceci suppose également des hypothèses restrictives, et l'on ne peut ainsi connaître que le nombre de gènes présentant précisément une dominance.

PANSE (I940) utilise chez des végétaux l'information apportée par la variation des variances à l'intérieur de familles produites par autofécondation en $F_{3}$, mais il n'apparaît pas clairement, semble-t-il, s'il estime la variation "vraie » des variances, déduction faite des fluctuations d'échantillonnage.

(1) On pourrait citer des travaux anciens comme ceux de Hays et Collaborateurs, cités par HuTr (1949), chez la poule, pour tenter d'interpréter la variation de plusieurs caractères de production dans cette espèce par l'intervention d'un ou deux gènes majeurs, mais ces tentatives reposaient sur une base théorique incertaine. 
Des méthodes plus récentes (WEHRHAHN et ALLARD, I965; ELSTON, I966) orientées dans la même optique générale, paraissent surtout pouvoir être utiles chez des espèces végétales où l'on dispose de lignées très homozygotes.

Une approche différente, toujours dans le cas où l'on dispose de populations relativement "pures » et de leurs croisements, est utilisée par PowERs (par exemple, PowERS, I950, I955, I963). Cet auteur teste des hypothèses génétiques particulières et relativement simples par la comparaison d'une distribution de fréquence à la combinaison, en proportion donnée, de plusieurs autres distributions connues; ainsi, dans l'hypothèse d'un gène majeur unique, la distribution d'un back-cross sera comparée à celle obtenue en mélangeant à part égale celles de $\mathrm{la}_{\mathrm{F}}$ et du parent correspondant ("Partitioning method )). Une idée analogue se retrouve dans le test plus élaboré proposé par STEWAR'T (I968).

Dans ce cas, la construction d'une hypothèse simple doit être suggérée par un premier examen des données $\left(\mathrm{F}_{2}\right.$ transgressant de façon importante l'intervalle de variation des lignées parentes, existence de plusieurs modes dans la distribution des $\mathrm{F}_{2}$ ou back-cross...); encore faut-il que les distributions de fréquences et les paramètres génétiques des diverses générations suggèrent l'hypothèse en question avec une netteté suffisante, ce qui semble, ici encore, devoir se limiter essentiellement au cas de lignées parentes fortement homozygotes.

Enfin, la comparaison de générations successives, soumises à une sélection accompagnée de back-cross répétés à une population initiale, peut fournir des indications dans cet ordre d'idées (ROBERTSON, I960, I964).

Nous avons proposé (MÉRAT, I968), pour la recherche de gènes à effet individuel important, cette fois en ségrégation à 1'intérieur d'une population unique, le test d'homogénéité des variances intra-familles, complété par l'examen de la normalité et des coefficients de forme (kurtosis et symétrie) des distributions à 1'intérieur des familles apparues les plus variables, comparées aux moins variables.

Pour toutes les méthodes mentionnées, y compris la nôtre, il est clair que les "facteurs " susceptibles d'être mis en évidence peuvent correspondre aussi bien à des régions chromosomiques d'étendue non négligeable qu'à des loci au sens strict du terme.

Le but du présent article est de montrer des exemples d'application de notre méthode à des populations de volailles.

\section{MATÉRIEL}

Plusieurs séries distinctes de données ont été utilisées :

$$
\text { I. - Troupeau de Jouy-en-Josas }
$$

La population étudiée est issue d'un croisement de plusieurs races fait en 1954 . Maintenue non fixée à divers loci de coloration ou à effet morphologique (MÉrAt, 1958, 1962, 1967 a) elle est soumise à un minimum de sélection pour les critères de production, et subdivisée en deux troupeaux reproduits l'un au printemps, l'autre en automne.

Tous les accouplements réalisés sont pedigree, avec un seul coq accouplé à une poule déterminée, et 8 à 10 poules par coq. A part le fait que les accouplements consanguins entre frères et 
scurs sont évités, il ne semble pas que le schéma de reproduction s'écarte beaucoup de la panmixic à l'intérieur de chaque sous-population dans laquelle notre méthode a été appliquée.

Le nombre de reproducteurs, à chaque génération et pour chaque sous-groupe considéré, est de 10 à 20 mâles et de 100 à 160 femelles.

Les jeunes sont élevés au sol jusqu'à 10 semaines d'âge. Les poulettes sont alors transférées en poulaillers de ponte, où leur production d'œufs est contrôlée au nid-trappe jusqu'à l'àge de 10 mois.

Les caractères quantitatifs étudiés ici sont ceux pour lesquels, a priori, ne se posait pas une question d'échelle telle qu'une corrélation entre moyenne et variance ou des bornes imposées à la variation. Ce sont le poids à 8 semaines pour chaque sexe, le nombre d'œufs produits de l'entrée en ponte au 31 décembre suivant (pour les animaux nés au printemps) ou à l'âge d'environ 10 mois (pour ceux nés à l'automne), le poids moyen des oufs par poule à 10 mois, le poids des poules et la longueur des barbillons au même âge; enfin, pour les dernières années, l'épaisseur moyenne des coquilles de deux oufs consécutifs pour chaque poule, mesurée au micromètre ( ${ }^{1}$ ).

Pour tous ces caractères, l'importance de la variabilité due au milieu est très appréciable, mais, les familles étant toutes distribuées dans les mêmes locaux d'élevage, il ne doit pas exister de facteurs importants de l'environnement plus variables pour certaines familles que pour d'autres. Ceci vaut également pour la date d'éclosion.

Les années étudiées vont de 1957 à 1967 inclus pour le poids à 8 semaines, le nombre d'cufs, leur poids moyen, le poids des poules adultes, et de 1964 à 1967 pour les autres caractères.

\section{2. - Troupeaux de la Station du Magneraud}

Nous avons examiné l'index des coquilles d'œufs (Moxgix, 1965) sur la souche "ponte" $M 99$ de type Rhode-Island pour les années 1964 à 1967.

D'autres données étaient constituées par les poids à 8 semaines de poulets issus de deux souches "chair" $X 77$ et $X 88$, maintenues sans sélection (Davaine Rouvier et Ricard, 1969). La souche $X 77$ était de type "Cornish", $X 88$ était issue de croisements entre la Bresse et d'autres races ("Bresse Pile").

Le schéma d'appariement des reproducteurs est analogue à celui du troupeau de Jouy, et la distribution des caractères étudiés ne s'écarte pas de façon importante, dans l'ensemble, de la normalité.

\section{3. - Poids d'organes}

Il s'agit de données recueillies par F. H. RiCARD, à la Station du Magneraud, sur les poids de divers organes de 315 poulets pedigree de 11 semaines d'âge, issus de 8 pères et de 32 mères. Ces animaux appartenaient à des lots expérimentaux réalisés en 1962, à partir de la souche "Bresse Pile " (Ricard et Rouvier, 1967). Les poids d'organes étaient exprimés en p. cent du poids corporel. Aucune transformation ultérieure n'était appliquée à ces données.

Pour ces données également, la distribution globale de chaque caractère ne s'écartait pas de façon caractérisée de la normalité, et la variance ne présentait pas de corrélation visible avec la moyenne.

\section{MÉTHODES}

Le fondement théorique de la méthode d'investigation a été exposé par ailleurs (MÉrAT, 1968). Deux tests sont utilisés, pour le cas de caractères à distribution continue :

1) Celui de l'homogénéité des variances intra-familles, valable du point de vue statistique si les distributions ne s'écartent pas ou peu de la normalité, notamment par dissymétrie, comme nous l'avions signalé. Quant aux écarts à la loi normale par aplatissement Box (1953) a montré qu'alors la probabilité réelle que le $\chi^{2}$ de Bartlett excède un niveau donné de signification était en fait inférieure à celle prévue.

Nous appliquons le test de Bartlett (Snedecor, 1959, p. 285) d'une part aux familles de même mère considérées intra-pères, de l'autre aux familles de même père, en caractérisant chacune par les variances intra-mères groupées pour l'ensemble de la famille. Dans le premier cas, un $\chi^{2}$ de Bartletr est calculé pour chaque père, et les $\chi^{2}$ relatifs aux différents pères sont cumulés, ainsi que leurs degrés de liberté, en vue d'un test global unique.

(1) Nous étudierons dans un autre article le cas du nombre de rectrices ayant mué à huit semaines, caractère à variation discontinue auquel notre méthode ne peut s'appliquer que d'une façon approchée.

Pour un caractère comme le nombre d'œufs, il arrive que la moyenne soit liée négativement avec la variance, et que la distribution soit dissymétrique avec un plus grand étalement vers les valeurs inférieures, cet étalement pouvant être dû en tout ou en partie à des facteurs du milieu. Cependant, ceci n'était que peu ou pas apparent dans les données présentes. 
Nous n'avons pas jugé nécessaire de considérer les données intra-lots d'éclosion, dans la mesure où la répartition de chaque famille dans ces lots (spécialement quant aux pères) n'apparaissait pas assez inégale pour pouvoir produire une hétérogénéité appréciable des variances.

Les variations d'effectif suivant les familles ne doivent pas, d'autre part, influer sur le résultat du test.

Dans tous les cas, les $\chi^{2}$ de BartLetr sont calculés intra-années, puis cumulés pour les différentes années, ainsi, d'autre part, que les degrés de liberté.

2) Celui de la normalité des distributions des écarts aux moyennes de familles et de la forme de ces distributions (kurtosis et symétrie : SNEDECOR, 1959), d'une part pour les familles ayant la variance observée la plus grande, de l'autre dans celles de plus petite variance, la séparation entre ces deux groupes étant faite arbitrairement (valeurs supérieures ou inférieures à la variance estimée sur l'ensemble des groupes). Nous avions indiqué (Mérat, 1968) que cette séparation en sous-groupes n'introduit pas a priori de biais dans l'estimation des coefficients de forme.

Le test de normalité est un simple $\chi^{2}$ d'ajustement d'une distribution normale aux distributions observées, subdivisées en classes; le nombre de celles-ci était le plus souvent de l'ordre de 10 à 20, les extrêmes étant 7 (certains poids d'organes) et plus de 40 (poids à 8 semaines). Les nombres de degrés de liberté dans les tableaux 1 et 2 sont égaux aux nombres de classes diminués de trois unités.

Quant aux coefficients $g_{1}$ et $g_{2}$, leur valeur est testée par rapport à zéro, caractéristique de la normale, comme nous l'avions décrit en détail auparavant (MÉRAT, 1968).

En complément du premier test, l'estimation d'héritabilité du caractère étudié peut fournir une indication appréciable. En effet, dans le cas où cette héritabilité est élevée, il est plus difficile d'interpréter une hétérogénéité importante des variances par l'existence de gènes de "sensibilité " au milieu, la variance " milieu " ou "génotype $\times$ milieu " ne pouvant pas, alors, dépasser une valeur déterminée par celle de l'héritabilité.

\section{RÉSULTATS}

Les tableaux I et 2 contiennent, pour les diverses séries de données, le résultat des tests d'homogénéité des variances intra-familles de père ou de mère intra-père, les tests de normalité de la distribution des écarts aux moyennes de familles de frères-sœurs respectivement parmi les familles présentant les plus grandes ou les plus petites variances, enfin l'indication des coefficients de forme (symétrie et kurtosis) des mêmes distributions dans ces deux groupes.

Nous n'indiquons au tableau I que les résultats sur l'ensemble des générations, mais la tendance à l'hétérogénéité des variances, lorsqu'elle existe, est en général marquée dans toutes les années, au moins pour les familles de même père. Ainsi, le $\chi^{2}$ de BARTLETT pour ces familles est significatif au moins au seuil $5 \mathrm{p}$. Ioo dans I 8 générations sur 22 pour le poids à 8 semaines dans chaque sexe, dans 20 pour le poids des + 우 adultes, dans Io pour le nombre d'œufs, dans 7 (sur ro) pour la longueur des barbillons.

Au total, les tableaux I et 2 contiennent $2 \mathrm{I}$ caractères soumis à chacun des tests successifs impliqués dans notre méthode. Il est intéressant de noter la fréquence des différents niveaux de signification obtenus sur ces $2 \mathrm{I}$ caractères pour chaque test.

Pour le test d'homogénéité des variances, les $\chi^{2}$ de BARTLETT " entre pères" sont 9 fois significatifs à $0, I$ p. IOO, I fois à I p. IOO, I fois à 5 p. IOO, et Io fois seulement ils n'atteignent pas ce dernier seuil. Quant aux $\chi^{2}$ de BARTLETT " entre mères intra-pères ", 9 sont significatifs au seuil o,I p. IOO, 5 à I p. IOO, 2 à 5 F. IOO. L'hétérogénéité des variances intra-familles est donc un fait assuré, et qui existe dans la majorité des cas étudiés. On peut le conclure malgré la difficulté introduite par certains écarts, d'ailleurs limités, à la normalité étant donnés les niveaux de signification élevés attachés aux $\chi^{2}$ calculés. 


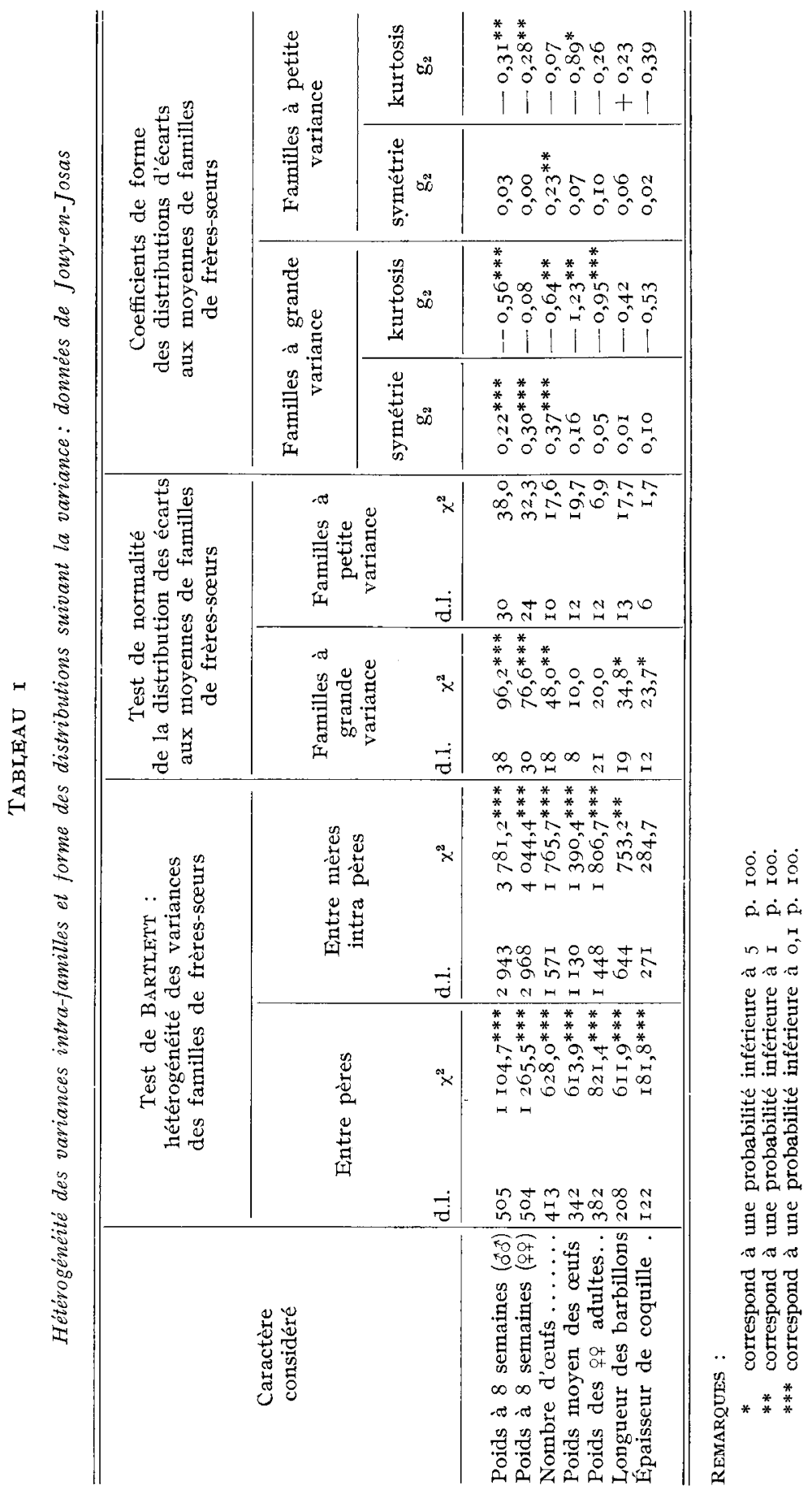


GÈNES MAJEURS

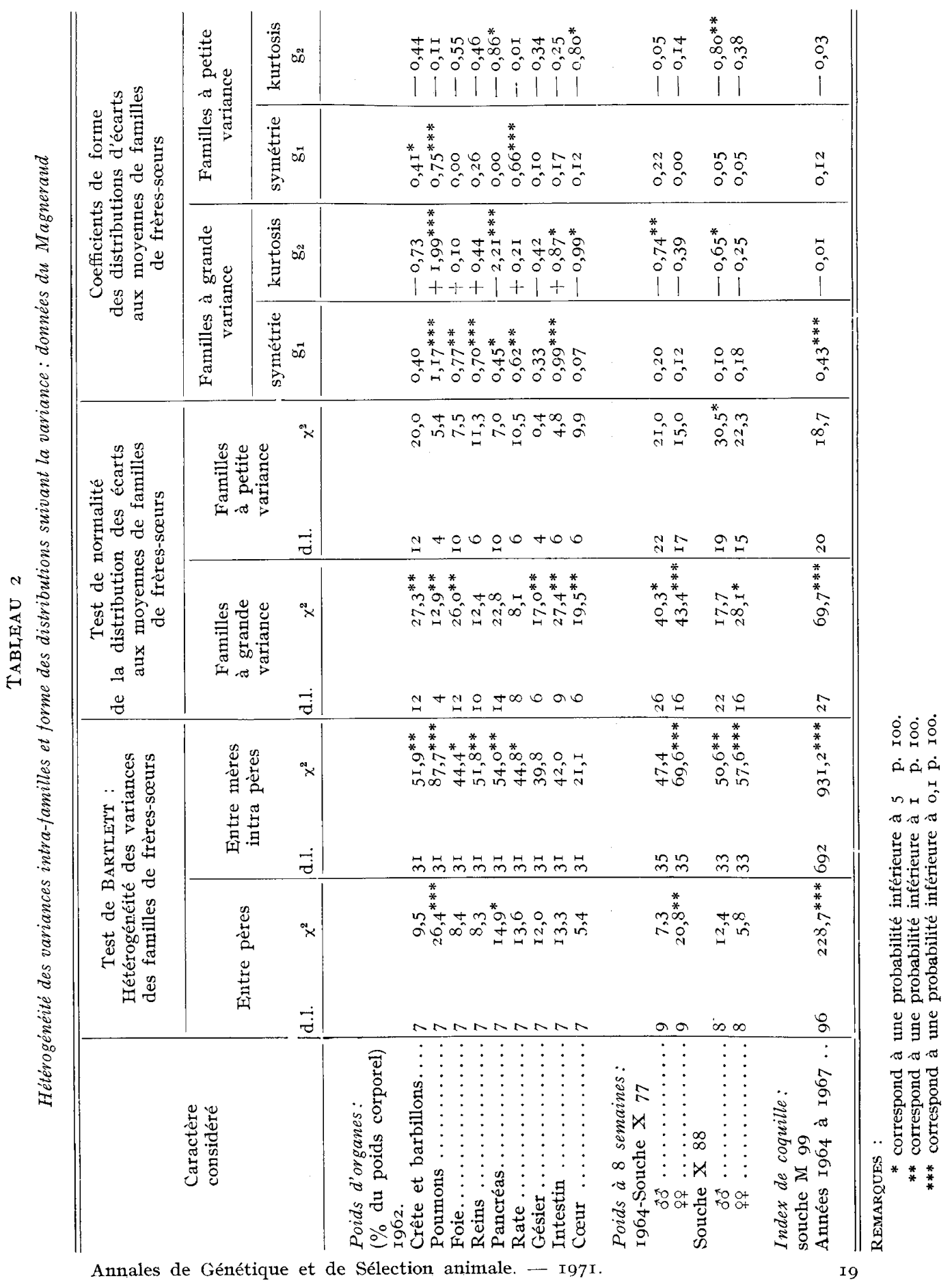




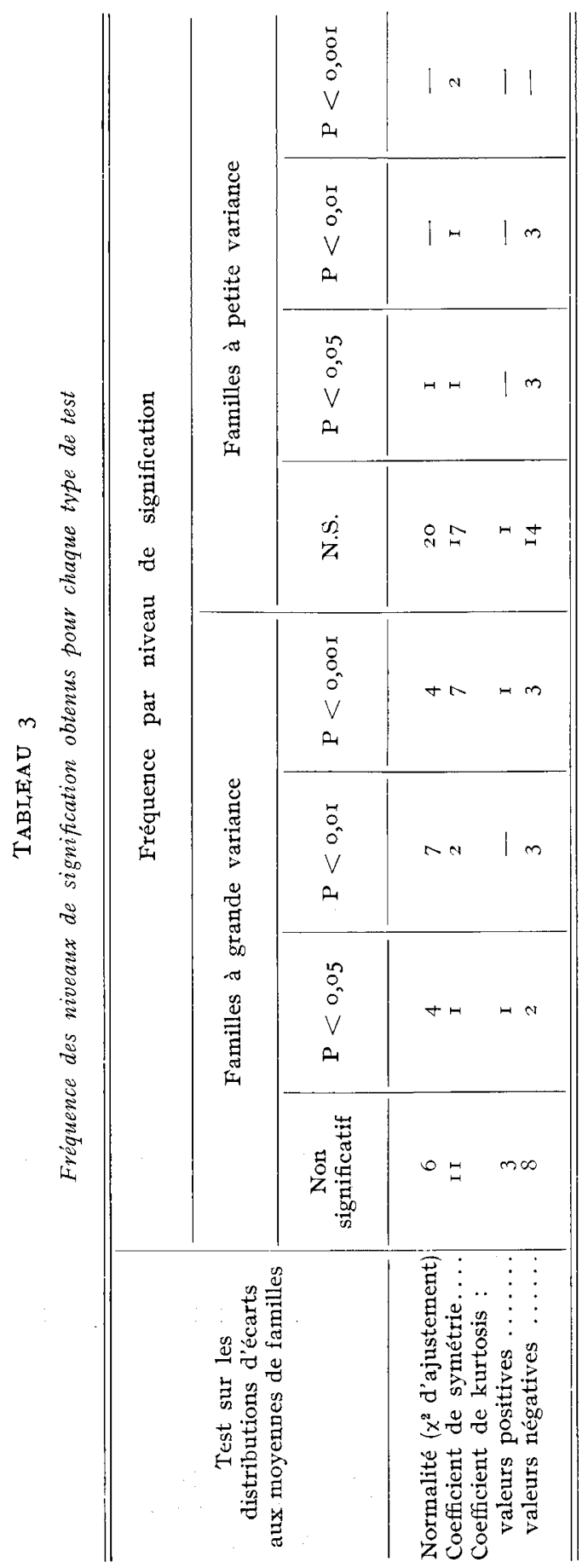


Pour les tests sur les distributions (normalité et test des coefficients de forme par rapport à la valeur zéro), le tableau 3 indique la fréquence des différents seuils de signification atteints.

Pour la normalité testée par un $\chi^{2}$ d'ajustement, la présence de distributions s'écartant de la loi normale parmi le groupe des familles "à grande variance " peut être considérée comme certaine, plus des deux tiers des tests étant dans ce cas significatifs au seuil 5 p. Ioo. Par contre, en ce qui concerne les familles " à petite variance ", la normalité est la règle, le seul test trouvé significatif au seuil $5 \mathrm{~F}$. Ioo parmi 2 I pouvant être le simple résultat d'un échantillonnage aléatoire. Au total, le test de normalité utilisé présente certaines limitations (BLISS, I967); mais les tendances qu'il indique paraissent assez claires.

Parallèlement, on constate que les coefficients tant de symétrie $\left(g_{1}\right)$ que de kurtosis $\left(\mathrm{g}_{2}\right)$ s'écartent de la valeur zéro, caractéristique de la loi normale, plus souvent dans les familles à grande variance. Dans quelques cas concernant les familles à petite variance, 1'un de ces coefficients s'écarte d'ailleurs significativement de zéro alors que le $\chi^{2}$ testant la normalité n'est pas significatif, ce qui reflète seulement une sensibilité assez faible de ce dernier test.

Pour le coefficient de kurtosis, en particulier, ce dernier est significatif au moins au seuil 5 p. Ioo et négatif (correspondant à un "aplatissement ") 8 fois sur 2 I parmi les familles de plus grande variance. Le signe négatif est en tout cas de loin le plus fréquent, y compris dans les familles à plus faible variance.

En fait, certains des caractères figurant au tableau 3 présentent entre eux une corrélation appréciable (poids à 8 semaine dans les deux sexes; poids des 우우 adultes et poids des œufs) et des résultats des tests les concernant ne sont pas indépendants. Cependant, si l'on supprime le poids à 8 semaines des mâles d'une part, le poids des femelles adultes de l'autre, les caractères restants peuvent pratiquement être considérés comme non liés, et l'on constate alors que, dans les familles à grande variance, sur I7 variables restantes, I3 présentent un écart significatif à la normalité, 9 un écart par asymétrie ( $g_{1}$ non nul), et 4 par aplatissement ( $g_{2}$ significativement négatif) : la conclusion générale reste la même.

\section{DISCUSSION ET CONCLUSIONS}

I) Le premier fait apparu avec évidence dans notre analyse est 1'hétérogénéité des variances suivant les familles (frères-sœurs ou demi-frères, demi-sœurs). Cette hétérogénéité a rarement été testée par d'autres auteurs; BRAY et al. (I960), qui la recherchaient entre groupes de poules sœurs ou demi-sœurs appartenant au même lot d'éclosion, l'ont eux aussi constatée. Il pourrait donc s'agir d'un fait général ou du moins fréquent.

Si cette hétérogénéité, dans les limites observées ici, ne met pas réellement en cause la validité des tests statistiques usuels, elle demande en tous cas à être interprétée sur le plan biologique. Comme nous l'avons indiqué dans l'article déjà cité (MÉRAT, I968), trois hypothèses peuvent être suggérées pour l'expliquer :

- Ségrégation de gènes différant suivant la famille par leur nombre ou l'importance de leurs effets. 
- Répartition variable, d'une famille à l'autre, de gènes de "sensibilité » à des fluctuations d'origine non génique.

- "Effets maternels" sur la variance des descendants.

La troisième hypothèse ne rendrait compte que d'une hétérogénéité entre familles de même mère. Par contre, aucune des deux autres ne peut être rejetée a priori. Comme nous l'avons signalé (MÉRAT, I968), la première est, strictement parlant, toujours vérifiée. Dans toute population non homozygote, il existe un facteur d'hétérogénéité de la variance suivant les familles, qui présentent une ségrégation pour des gènes différents. Si un nombre limité de loci est responsable d'une fraction importante de la variance totale, cette hétérogénéité sera appréciable, mais nous avons cependant indiqué qu'un nombre de loci de l'ordre de plusieurs dizaines peut encore avoir un effet non négligeable dans ce sens.

Quant à la présence de différences génétiques de "sensibilité au milieu ", elle sera d'autant Flus plausible a priori pour expliquer les hétérogénéités de variances observées que le milieu, précisément, aura une grande part dans la variabilité du caractère étudié. On peut seulement noter que 1'hétérogénéité des variances intrafamilles concerne, dans nos données, aussi bien ou davantage des caractères d'héritabilité généralement assez élevée (poids d'œufs, poids adultes ..) que d'autres.

2) Plus significatifs pour notre propos sont les écarts à la normalité, fréquents dans les familles les plus dispersées et pratiquement absents des autres, dans les limites de sensibilité du test. On paut toutefois distinguer entre les écarts à la normalité par dissymétrie ou par aplatissement.

Concernant la symétrie, on pourrait concevoir, dans certains cas, des gènes répondant de façon dissymétrique à des aléas du milieu, par exemple uniquement dans un sens défavorable à la performance considérée.

Nous en avons un indice dans le cas des index de coquilles de la souche M 99 au Magneraud. Sur des données de 1967 de cette souche, nous avons obtenu les variances intra-poule de ces index, basées sur 5 œufs consécutifs par poule. En comparant ces variances, évidemment de nature non génétique, chez des familles à " petite ", " grande " ou " très grande " variance entre moyennes individuelles intrafamille (les limites entre groufes sont arbitraires, celle entre les deux premiers correspondant à la variance estimée sur l'ensemble des familles), on obtient :

— pour les premières : $s^{2}=0,029$ avec 6360 degrés de liberté

- pour les secondes : $s^{2}=0,030$ avec 2476 degrés de liberté

- pour les troisièmes : $s^{2}=0,034$ avec I 832 degrés de liberté

La différence entre le dernier groupe et les deux autres, quoique légère, est hautement significative. Ici, 1'hétérogénéité des variances intra-familles pourrait s'expliquer au moins partiellement par une différence de la variance non génétique, cette différence étant conditionnée génétiquement.

3) La situation est différente en ce qui concerne le coefficient de kurtosis, notamment et à plus forte raison lorsque le coefficient de symétrie ne s'écarte pas de zéro, comme dans le cas du poids des œufs, du poids des poules adultes, du poids à 8 semaines dans les souches X 77 et X 88 chez les mâles, de certains poids d'organes. 
Comme nous l'avions discuté (MÉRAT, I968), on ne conçoit guère que l'intervention d'un nombre limité de gènes à effet important, en ségrégation dans certaines familles et nor dans d'autres, comme possibilité vraisemblable pour expliquer des coefficients de kurtosis significativement négatifs (distributions aplaties) dans les familles les plus variables.

Or, comme le tableau 3 l'a montré, le signe négatif est de loin prépondérant parmi les coefficients de kurtosis estimés ici. Il est vrai que ceci s'observe non seulement dans les familles à grande variance mais aussi dans les autres; toutefois, les cas de coefficients significativement négatifs sont nettement plus nombreux et plus extrêmes dans les premières, et il faut par ailleurs tenir compte de ce que notre séparation entre familles à " grande " et à " petite " variance étant parfaitement arbitraire, ces dernières peuvent aussi, éventuellement, comporter des ségrégations de gènes d'effet individuel appréciable.

Ces résultats suggèrent donc la présence, pour une proportion notable des cas étudiés, d'un nombre limité de gènes ou régions chromosomiques rendant compte d'une fraction importante de la variance totale. Si imprécis soit-il, cet indice vaut d'être signalé. Bien entendu, notre méthode n'indique pas, pour tel ou tel caractère, si, par exemple, 50 ou 80 p. Ioo de la variance génétique totale sont déterminés par 2 ou 3 loci, ou par I 5 ou 20 , les conséquences pratiques pouvant être différentes dans les deux cas. Nous présenterons cependant, dans une note séparée, une tentative d'estimation très grossière du nombre de loci, en les supposant tous d'effet équivalent : les ordres de grandeurs suggérés sont, dans 1'ensemble, compris entre les valeurs qui viennent d'être indiquées.

Le seul autre travail utilisant la même méthode, à notre connaissance, est celui de Hammond et James (I970), Pour deux caractères (nombre de soies abdominales et nombre de descendants) dans une population panmictique de drosophiles, aucune présence de gène " majeur " n'était suggérée, le coefficient de kurtosis en particulier n'étant pas flus négatif dans les familles à grande variance que dans les autres. Cependant, les meilleures conditions n'étaient peut-être pas réalisées pour une telle analyse. Le test de BARTLETT n'indiquait pas une hétérogénéité significative des variances intra-familles, condition que nous avions supposée dans l'application de notre méthode. De plus, les auteurs font remarquer qu'il s'agit de caractères à héritabilité faible dans la population étudiée. Ceci peut contribuer à expliquer la différence avec nos résultats, car la plupart des caractères que nous étudions ont une héritabilité moyenne ou relativement élevée (de 1'ordre de $0,4$ à 0,6$)$ notamment pour les poids à différents âges, les épaisseurs de coquille, le poids des œufs. Il en est probablement de même du poids d'organes (données non publiées).

En fin de compte, les résultats présents sont au moins un encouragement à étendre cette recherche. Nous comptons présenter ultérieurement des données relatives à des caractères à variation non continue comme l'emplumement à 8 semaines (nombre de rectrices ayant mué), l'extension du noir dans le plumage, le nombre de crêtillons, pour lesquels une méthode dérivée de celle utilisée ici paraît confirmer la présence, déjà "soupçonnée, d'un très petit nombre de gènes majeurs. 


\section{REMERCIEMENTS}

Nous remercions L. P'. Cochez et F. H. Ricard, Station expérimentale d'Aviculture du Magneraud, d'avoir mis à notre disposition des données qui constituent une partie importante de cet article.

Nous exprimons également notre reconnaissance au $\mathrm{D}^{\mathrm{r}} \mathrm{G}$. A. Cisayton, Institute of Animal Genetics, Edinburgh, et au Dr K. Hammond, Université de Sydney, pour leur lecture de ce manuscrit et leurs critiques et remarques constructives.

\section{SUMMARY}

\section{FREQUENCY DISTRIBUTIONS, INTERPRETATION OF THE GENETIC FACTORS GOVERNING THE TRANSMISSION OF THE QUANTITATIVE CHARACTERS AND DETERMINATION OF “ MAJOR" GENES : EXPERIMEN'TAL DATA.}

We looked for large-effect genes in several pedigree poultry populations at the Jouy-en-Josas and Le Magneraud Stations, and for various quantitative traits having an over-all distribution not deviating much from the normal. These traits were body weight, egg number, egg traits, and organ weights. A method previously proposed by MERAT (1968) was used. This consisted of test of the form (asymmetry, kurtosis) of distributions of deviations from family averages, separately for families with the higher vs. the lower observed variance, when heterogeneity of these intrafamily variances has been shown by a preliminary test.

In a high percentage of cases, intrafamily variances are very significantly heterogenous. On the other hand, the distribution of deviations from family averages is often non-normal for , higher variance " families, and practically never for the other ones, within the limits of test sensitivity. Departures from zero for asymmetry and kurtosis coefficients are more often significant and more extreme in the , higher variance" group. Finally, kurtosis cocfficients are, for the most part, negative.

In an appreciable number of cases on the whole, these results suggest the presence of a limited number of genes or chromosomal regions accounting for an important part of the total genetic variance. They are thus an encouragement to continue this research:

\section{RÉFÉRENCES BIBLIOGRAPHIQUES}

Burss C. I., 1967. Statistics in Biology. Mc Graw Hill, New York.

Box G. F. P., I953. Non-normality and tests on variances. Biometrika, 40, 318-335.

BRAY D. F., KIng S. C., ANDERson V. I., I960. Sexual maturity and the measurement of egg production. Poult. Sci. 39, 590-60г.

CASTLE W. E., I92 I $a$. On a method of estimating the number of genetic factors concerned in cases of blending inheritance. Science 54, 93-96.

CASTLE W. E., I92 I $b$. An improved method of estimating the number of genetic factors concerned in cases of blending inheritance. Science, 54, 223.

CASTLE W. E., I940. Influence of certain color mutants on body size in mice, rats and rabbits. Genetics, 26, I77-19r.

Charles O. R., Goodwin R. H., I943. An estimate of the minimum number of genes differentiating two species of golden rod with respect to their morphological characters. Amer, Nat., 77, 53-69.

DAvarne P., Rouvier R., Ricard F. H., 1969. Relations entre les expressions de la variabilité morphologique intra et entre familles chez le poulet. Biométrie Praximétrie, 10, 3-2I.

Dempster E. R., SNyder I. A., I950. A correction for linkage in the computation of number of gene differences. Science, 111, $283-285$.

Fist E. M., rgr6. Inheritance in crosses betwcen Nicotiana Langsdorfi and Nicotiana Alata. Genetics, 1, 3 I I-333.

FisTON R. C., I966. On testing whether one locus can account for the genetic difference in susceptibility between two homozygous lines. Genetics, 54, 89-94.

FISHER R. A., ImMer F. R., Tedin O., I932. The genetical interpretation of statistics of the third degree in the study of quantitative inheritance. Genetics, 17, I07-124. 
Gilmour D. G., I960. Blood groups in chickens. Brit. Poult. Sci., 1, 75-100.

Hammond K., James J. W., I97o. Genes of large effect and the shape of the distribution of a quantitative character. Aust. J. biol. Sci., 23, 867-876.

Hutr F. B., I949. Genetics of the fowl. Mc Graw Hill, New-York.

Mather K., 1949. Biometrical Genetics. Methuen et Co, London.

Merat P., I958. Génétique factorielle et production chez les volailles. I. Plumage "herminé " ou "sauvage ": Relation avec la croissance en poids. Annls Zootech., 7, I23-142.

Merat P., I962. Quelques relations entre caractères extérieurs à hérédité simple et productivité. XIIth World's Poultry Congress, 1, $7 \mathrm{r}-76$.

Merat P., I967 a. Contribution à l'étude de la " valeur sélective " associée à quelques gènes chez 1? poule domestique. I. Différences quantitatives liées au génotype individuel. Ann. Biol. Anim. Bioch. Biophys. 7, 79-104.

Merat P., I $967 \mathrm{~b}$. Contribution à l'étude de la "valeur sélective " associée à quelques gènes chez la poule domestique. Ir. Effets maternels. Ann. Biol. Anim. Bioch. Biophys., 7, I83-203.

Merat P., I968. Distributions de fréquences, interprétation du déterminisme génétique des caractères quantitatifs et recherche de "gènes majeurs ". Biometrics, 24, 277-293.

Mongrn P., I965. Index de solidité de coquille de l'œuf. Ses significations. Sa précision. Ann.Zootech., 14, $319-325$.

Panse V. G., r940. Application of genetics to plant breeding. II. The inheritance of quantitative characters and plant breeding. J. Genet., 40, 283-302.

Powers L., I950. Partitioning method of genetic analysis applied to quantitative characters of tomato crosses. U.S.D.A. Rech. Bull., 998.

Powers L., I955. Components of variance method and partitioning method of genetic analysis applied to weight per fruit of tomato hybrid and parental populations. U.S.D.A. Tech. Bull., II 3-I.

Powers $L_{1 .}$, I963. The partitioning method of genetic analysis and some aspects of its applications to plant breeding. Statistical genetics and plant breeding. Nat. Acad. Sci., Natl. Res. Council Publ. 982, $280-318$.

Ricard F. H., Rouvier R., I967. Étude de la composition anatomique du poulet. I. Variabilité de la répartition des différentes parties corporelles chez des coquelets "Bresse-pile ". Annls Zoot., 16, 23-39.

Robertson A., I96o. A theory of limits in artificial selection. Proc Roy. Soc. B., 153, 234-249.

RoBertson A., I964. The effect of initial reverse selection upon total selection response. Genet. Res., 5, 68-79.

SAX K., I923. The association of size differences with seedcoat pattern and pigmentation in phaseolus vulgaris. Genetics, 8, 552-560.

Serebrovsky A. S., I928. An analysis of the in heritance of quantitative transgressive characters. $Z$. indukt. A bst. Veverb-Lehre, 48, 229-243.

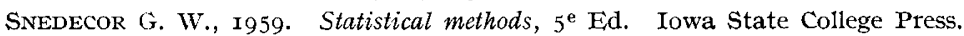

SpIckett S. G., I963. Genetic and developmental studies of a quantitative chatacter. Nature, 199, $870-873$.

SpIcketr S. G., Thoday J. M., r966. Regular responses to selection 3. Interaction between located polygenes. Genet. Res., 7, 96-121.

STEWART J., I969. Biometrical genetics with one or two loci. I. The choice of a specific genetic model. Heredity, 24, 2 II-224.

Thoday J. M., I96r. Location of polygenes. Nature, 191, 368-37o.

Thoday J. M., Gibson J. B., Spickett S. G., I964. Regular responses to selection. 2. Recombination and accelerated response. Genet. Res., 5, I-ז9.

Wehrhahn C., Allard R. W., 1965. The detection and measurement of the effects of individual genes involved in the inheritance of a quantitative character in wheat. Genetics, 51, rog-I I9.

WrIGHT S., r927. The effects in combination of the major color factors of the guinea pig. Genetics, 12, 530-569.

WrIGHT S., 1952. The genetics of quantitative variability. In quantitative inheritance, London, Publ. Agric. Res. Coun, 5-4I. 